\title{
Large-scale magnetic fields of low-mass dwarfs: the many faces of dynamo
}

\author{
J.-F. Donati \\ CNRS/Université de Toulouse, Observatoire Midi-Pyrérées, Toulouse, France \\ email: donati@ast.obs-mip.fr
}

\begin{abstract}
Magnetic field are ubiquitous to low-mass stars and can potentially impact their evolution and their internal structure; yet the physical processes (called dynamo) that succeed at generating them in the stellar convective zones of cool dwarfs are still enigmatic. Although theoretical modelling and numerical simulations of stellar dynamo action showed breathtaking progress in the last decade, they are not yet in the state of accurately predicting the various magnetic topologies that different low-mass stars can generate.

Thanks to the advent of new-generation instruments, spectropolarimetric observations can now reveal the large-scale magnetic topologies of cool dwarfs, from the brown dwarf threshold (spectral type M8) up to the limit beyond which outer convective zones get vanishingly small (spectral type F5). In particular, they can reconstruct through tomographic methods the poloidal and toroidal components of the large-scale field, hence offering a fresh option for guiding dynamo theories to a more mature state.

We review here the latest observational advances, showing in particular that magnetic topologies of low-mass dwarfs can drastically vary with mass and rotation rate, and discuss their implications for our understanding of dynamo processes.
\end{abstract}

Keywords. stars: magnetic fields, stars: imaging, stars: rotation, stars: late-type, techniques: polarimetry

\section{Introduction}

Most cool stars exhibit a large number of solar-like activity phenomena; dark spots are present at the their surfaces (e.g., Berdyugina 2005), where they come and go on timescales ranging from days (as they are carried in and out of the observer's view by the star's rotation) to months (as they appear and disappear over a typical spot lifetime) and years or decades (with spots fluctuating in number and location throughout activity cycles). Prominences are also detected in cool stars, both as absorption and emission transients (e.g., in Balmer lines) tracing magnetically confined clouds (e.g., Cameron \& Robinson 1989) either transiting the stellar disc (and scattering photons away from the observer, as for dark filaments on the Sun) or seen off-limb (and scattering photons towards the observer, as for bright prominences on the Sun). Cool stars are also surrounded by low-density coronal plasma at MK temperatures showing up at various wavelengths in the spectrum (e.g., radio, X-ray and optical line emission) and associated with frequent flaring, recurrent coronal mass ejections, and winds. Activity phenomena in cool stars scale up with faster rotation and later spectral types (e.g., Hall 2008).

The current understanding is that activity phenomena are a by-product of the magnetic fields that cool stars generate within their convective envelopes through dynamo processes, involving cyclonic turbulence and rotational shearing. In the particular case of the Sun, dynamo processes are presumably concentrating in a thin interface layer (the so-called tachocline) confined at the base of the convective zone (CZ) and where rotation gradients are supposedly largest. The spectacular images of the Sun collected with 
TRACE and (more recently) HINODE demonstrate that the activity of the Sun very tightly correlates with the presence of magnetic field emerging from the surface, either in the form of large closed loops (mostly at medium latitudes) or open field lines (mostly at high latitudes); the exact process through which magnetic fields succeed at heating the tenuous outer atmosphere to MK temperatures is however still unclear.

Cool stars are assumed to behave similarly. This is supported by observations showing that activity scales up with rotation rate (at any given spectral type), as suggested by dynamo theories. One of the key parameter for measuring the efficiency of magnetic field generation is the Rossby number Ro, i.e., the ratio of the rotation period of the star to the convective turnover time. It describes how strongly the Coriolis force is capable of affecting the convective eddies, with small $R o$ values indicating very active stars rotating fast enough to ensure that the Coriolis force strongly impacts convection. The observation that activity correlates better with Ro than with rotation (e.g., Noyes et al. 1984, Mangeney \& Praderie 1984) or equivalently, that cooler stars are relatively more active at a given rotation rate, agrees well with the theoretical expectation that convective turnover times increase with decreasing stellar luminosities.

Magnetic fields are also responsible for slowing down cool stars through the braking torque of winds magnetically coupled to the stellar surface (Schatzman 1962; Mestel 1999). This is qualitatively compatible with the fact that most cool stars rotate slowly (like the Sun itself), with the exception of close binaries (whose spin angular momentum is constantly refueled from the orbital reservoir through tidal coupling) and young stars (which have not had time yet to dissipate their initial load of angular momentum). Magnetised wind models yield a good match to the observed distribution of rotation periods in young open clusters of ages ranging from several tens to several hundreds of Myr, further confirming that magnetic fields are likely what triggers the spinning down of cool stars as they arrive on the main sequence.

The main lesson from the solar paradigm is thus that dynamo processes are essentially ubiquitous in all cool stars with outer convective layers and generate magnetic fields with a high degree of temporal variability at all timescales. Extrapolating the solar analogy much further is potentially hazardous; in particular, assuming that conventional dynamo models (entirely tailored to match observations of the Sun) also apply to cool stars with very different convective depths and rotation rates is subject to caution. In very active stars rotating 100 times faster than the Sun for instance, the magnetic feedback onto the convection pattern may be strong enough to distort theoretical dynamo patterns; similarly, very-low-mass fully-convective stars obviously lack the interface layer where conventional dynamo processes are expected to concentrate, but are nevertheless strongly active. Magnetic studies of low-mass stars are therefore our best chance for exploring the various faces of dynamo processes over a large range of masses and rotation rates.

\section{Magnetic properties of cool stars}

The very first estimates of magnetic fields in cool stars other than the Sun were obtained by measuring the differential broadening of spectral lines as a function of their magnetic sensitivities (Robinson et al. 1980), making it possible to derive the first trends on the magnetic properties of low-mass stars (e.g., Saar 2001). These studies find that the average surface magnetic strength is, in most cases, roughly equal to the equipartition field, i.e., the field whose magnetic pressure balances the thermal pressure of the surrounding gas; only very active stars with rotation periods lower than about $5 \mathrm{~d}$ (among which fully-convective $\mathrm{M}$ dwarfs and young low-mass protostars) strongly deviate from this relation (e.g., Johns-Krull \& Valenti 1996) A similar behaviour is observed in the 
Sun, where fields of moderately active plages are close to equipartition while those of active sunspots are stronger by a factor of 2 or more. This suggests that magnetic regions at stellar surfaces progressively evolve from a plage-like to a spot-like structure, with flux tubes having increasingly larger sizes or being more tightly packed, as activity increases.

These studies also find that the average magnetic flux at the surfaces of cool stars increases more or less linearly with $1 / R o$ until it saturates at $R o \simeq 0.1$ (corresponding to a rotation period of about $2 \mathrm{~d}$ for a Sun-like star), with most of the increase being attributable to the fractional area covered with fields (at least in moderately active stars). The detection of a saturation regime, confirmed with new magnetic flux measurements from molecular lines in M dwarfs (Reiners \& Basri 2008), supports the idea that magnetic fields are eventually capable of modifying, if not controlling, the convective motions through some feedback mechanism; this may potentially explain in particular why magnetic regions at low and high activity levels are morphologically different.

The first detections of Zeeman polarisation signatures from solar-type stars (Donati et al. 1997) and their tomographic modelling with stellar surface imaging tools such as Zeeman-Doppler Imaging (or ZDI, e.g., Donati et al. 1992) opened up an alternative option for studying dynamo processes. In particular, the medium- and large-scale magnetic fields accessible through ZDI, though energetically less important than magnetic fluxes derived from Zeeman broadening, are nevertheless optimally suited for checking topological predictions of dynamo models on global fields and their potentially cyclic variations, to which other methods are insensitive.

Initial studies, concentrating on a few very active rapidly rotating stars in the saturateddynamo regime brought surprising results. In particular, they demonstrated that strong toroidal fields can show up directly at the stellar surface, in the form of monopolar regions of dominantly azimuthal fields or even complete rings encircling the star at various latitudes (Donati et al. 1992, Donati \& Cameron 1997, Donati 1999, Donati et al. 2003a); while tori of strong azimuthal fields are likely present in the Sun at the base of the CZ (e.g., to account for the non-stochastic arrangement of surface sunspots, known as Hale's polarity law), they do not build up at the surface of the Sun - hence the surprise. The poloidal components detected on the active stars observed in these exploratory studies consist mainly of a significant non-axisymmetric term with alternating patterns of opposite radial field polarities. Other studies confirmed and amplified these initial results, reporting the presence of strong and often dominant toroidal fields at photospheric level (Dunstone et al. 2008), even in less active stars with longer rotation periods (Petit et al. 2005) or earlier spectral types (Marsden et al. 2006). A recent study focussing on mainsequence Sun-like stars with different rotation periods suggests that significant surface toroidal fields are detected whenever the rotation period is lower than $\simeq 20 \mathrm{~d}$ (Petit et al. 2008 ), i.e., $\simeq 25 \%$ shorter than the rotation period of the Sun.

ZDI observations also demonstrated that large-scale magnetic topologies of active stars are latitudinally sheared by surface differential rotation at a level comparable to that of the Sun (Donati \& Cameron 1997), with the equator lapping the pole by one complete rotation cycle about every $100 \mathrm{~d}$. This conclusion agrees with previous results derived from indirect tracers of differential rotation (photometric monitoring, e.g., Hall 1991). Differential rotation displays a steep increase with earlier spectral types, reaching values of 10 times the solar shear or more in late F stars (e.g., Marsden et al. 2006, Donati et al. 2008a). This trend is independently confirmed from observations of spectral line shapes (Reiners 2006) and suggests that F stars with shallow convective zones (CZs) are departing very strongly from solid-body rotation.

The major improvement in instrumental sensitivity brought by the twin new-generation spectropolarimeters ESPaDOnS@CFHT and NARVAL@TBL made it possible to start 
surveying the magnetic topologies of cool stars, from mid $\mathrm{F}$ to late $\mathrm{M}$ stars. It allowed in particular the large-scale field properties of $\mathrm{M}$ dwarfs to be investigated for the first time on both sides of the full convection threshold (presumably occurring at spectral type M4, i.e., at a mass of $0.35 \mathrm{M}_{\odot}$, Baraffe et al. 1998). Spectropolarimetric monitoring of the rapidly rotating M4 dwarf V374 Peg revealed that the star hosts a strong large-scale mostly-poloidal, mainly axisymmetric field despite its very short period (0.44 d), high activity level and low Ro (Donati et al. 2006, Morin et al. 2008a); additional observations of active mid-M dwarfs further confirmed that dynamo processes in fully-convective stars with masses of about $0.3 \mathrm{M}_{\odot}$ are apparently very successful at generating strong poloidal fields with simple axisymmetric configurations (Morin et al. 2008b).

Comparing to partly-convective early $\mathrm{M}$ dwarfs reveals that the transition in the largescale field properties is fairly sharp and located at a mass of about 0.4 to $0.5 \mathrm{M}_{\odot}$ (Donati et al. 2008b), i.e., slightly above the $0.35 \mathrm{M}_{\odot}$ theoretical full-convection threshold. This sharp transition also coincides with a strong decrease in surface differential rotation (with photospheric shears smaller by a factor of 10 or more than that of the Sun) and with a strong increase in the lifetime of large-scale fields. Preliminary results on verylow mass stars $\left(<0.2 \mathrm{M}_{\odot}\right)$ suggest that the situation is even more complex, with some stars hosting very strong and simple large-scale fields (like those of mid-M dwarfs) and some others with much weaker and complex magnetic topologies (resembling those of early-M dwarfs). Observations of a larger sample are needed to clarify the situation but the preliminary results already demonstrate that at least some very-low-mass stars are capable of producing a strong large-scale axisymmetric poloidal field. This conclusion is independently confirmed by the detection of highly-polarised rotationally-modulated radio emission from late $\mathrm{M}$ and early $\mathrm{L}$ dwarfs attributable to intense large-scale magnetic fields (e.g., Berger et al. 2005) through electron cyclotron maser instability (Hallinan 2008).

Figure 1 presents graphically the main results obtained so far in the framework of the ongoing survey effort, aimed at identifying which stellar parameters mostly control the field topology. To make it more synthetic, the plot focusses only on a few basic properties of the reconstructed magnetic topologies, namely the reconstructed magnetic energy density $e$ (actually the integral of $B^{2}$ over the stellar surface), the fractional energy density $p$ in the poloidal field component, and the fractional energy density $a$ in mostly axisymmetric modes (i.e., with $m<\ell / 2, m$ and $\ell$ being the order and degree of the spherical harmonic modes describing the reconstructed field). Each selected star is shown in the plot at a position corresponding to its mass and rotation period, with a symbol depicting these three characteristics of the recovered large-scale fields, i.e., $e$ (symbol size), $p$ (symbol colour) and $a$ (symbol shape). This plot clearly illustrates the two main transitions mentioned above:

- below $R o \simeq 1$, stars more massive than $0.5 \mathrm{M}_{\odot}$ succeed at producing a substantial (and sometimes even dominant) toroidal component with a mostly non-axisymmetric poloidal component;

- below $0.5 \mathrm{M}_{\odot}$, stars (at least very active ones) apparently manage to trigger strong large-scale fields that are mostly poloidal and axisymmetric.

At very low-masses and high rotation rates, dwarfs seem capable of generating either strong aligned dipoles or very weak non-axisymmetric large-scale fields (Morin et al. 2010).

Long-term monitoring of large-scale magnetic topologies can potentially reveal whether the underlying dynamo processes are cyclic like in the Sun (with the field switching its overall polarity every $11 \mathrm{yr}$ ), constant or chaotic. Initial studies carried over a decade demonstrated indeed that both the field topologies and the differential rotation patterns 


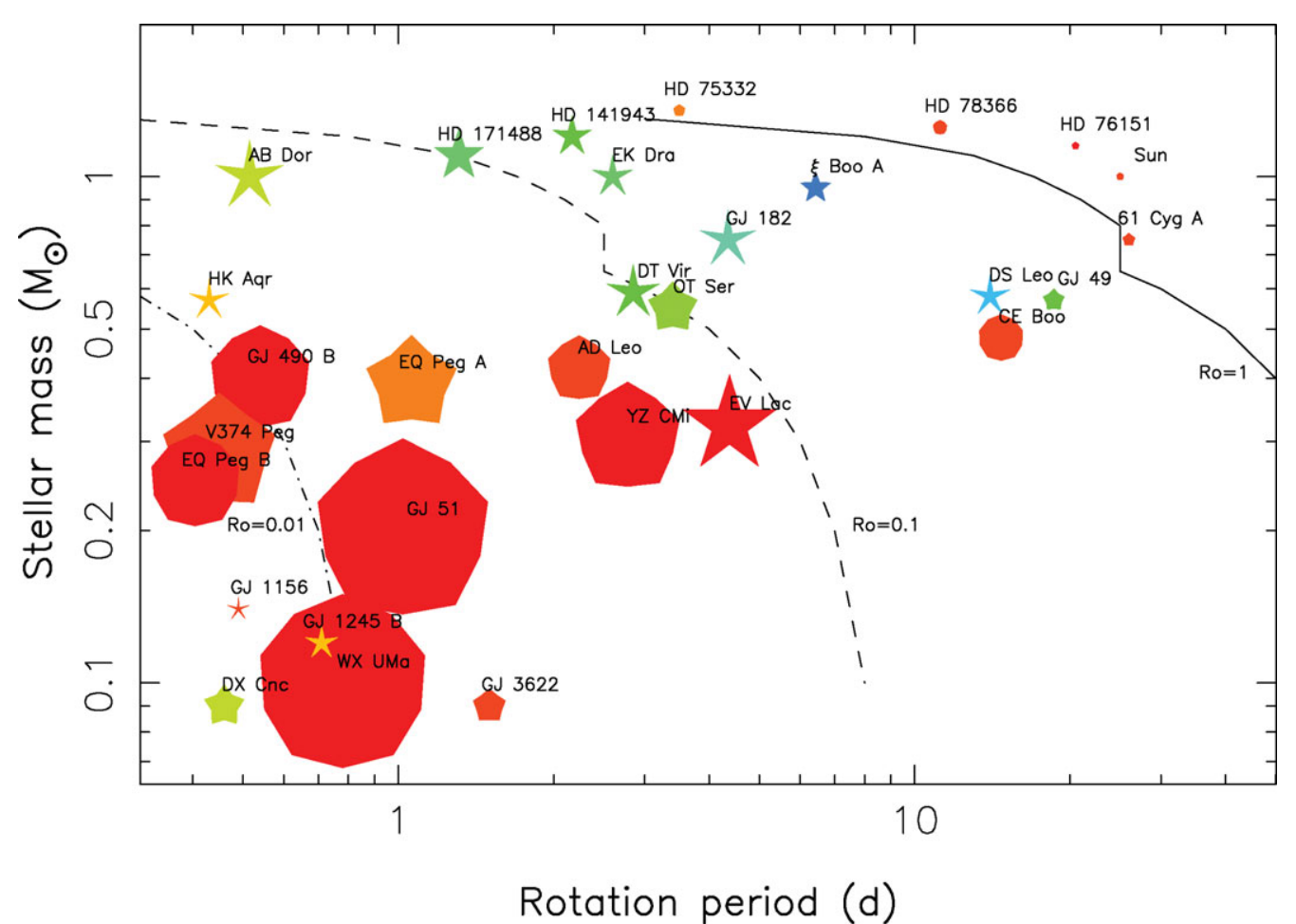

Figure 1. Basic properties of the large-scale magnetic topologies of cool stars, as a function of stellar mass and rotation rate. Symbol size indicates relative magnetic energy densities $e$, symbol colour illustrates field configurations (blue and red for purely toroidal and purely poloidal fields respectively) while symbol shape depicts the degree of axisymmetry of the poloidal field component (decagon and stars for purely axisymmetric and purely non-axisymmetric poloidal fields respectively). The full, dashed and dash-dot lines respectively trace where the Rossby number Ro equals 1, 0.1 and 0.01 . The smallest and largest symbols correspond to mean large-scale field strengths of $3 \mathrm{G}$ and $1.5 \mathrm{kG}$ respectively (updated from Donati \& Landstreet 2009).

are variable on long-timescales (e.g., Donati et al. 2003b) but have failed to catch stars in the process of switching their global magnetic polarities, suggesting that their dynamos (if cyclic) do not reverse much more often than that of the Sun; similar conclusions are obtained from long-term monitoring of solar-type stars using indirect proxies like overall brightness or chromospheric emission (Hall 2008). Very recently, first evidence for global polarity switches was reported in a star other than the Sun, namely the Jupiterhosting F8 star $\tau$ Boo (Donati 2008a). During repeated spectropolarimetric monitoring (see Fig. 2), two successive polarity switches of $\tau$ Boo were recorded within about 2 yr, suggesting an activity cycle about 10 times faster than that of the Sun (Fares et al. 2009); although still fragmentary, observations already show that the poloidal and toroidal field components do not vary in phase across the cycle period.

\section{Testing dynamo models with observations}

Observational evidence that magnetic fields of cool stars are generated through dynamo processes is very strong. As recalled above, magnetic fields are ubiquitous to all stars with significant outer convection (i.e., spectral type later than mid F), and direct spectroscopic estimates demonstrate that magnetic fluxes scale up with rotation rate (and more tightly with $1 / R o$ ) until they saturate - in agreement with what conventional dynamo theories 

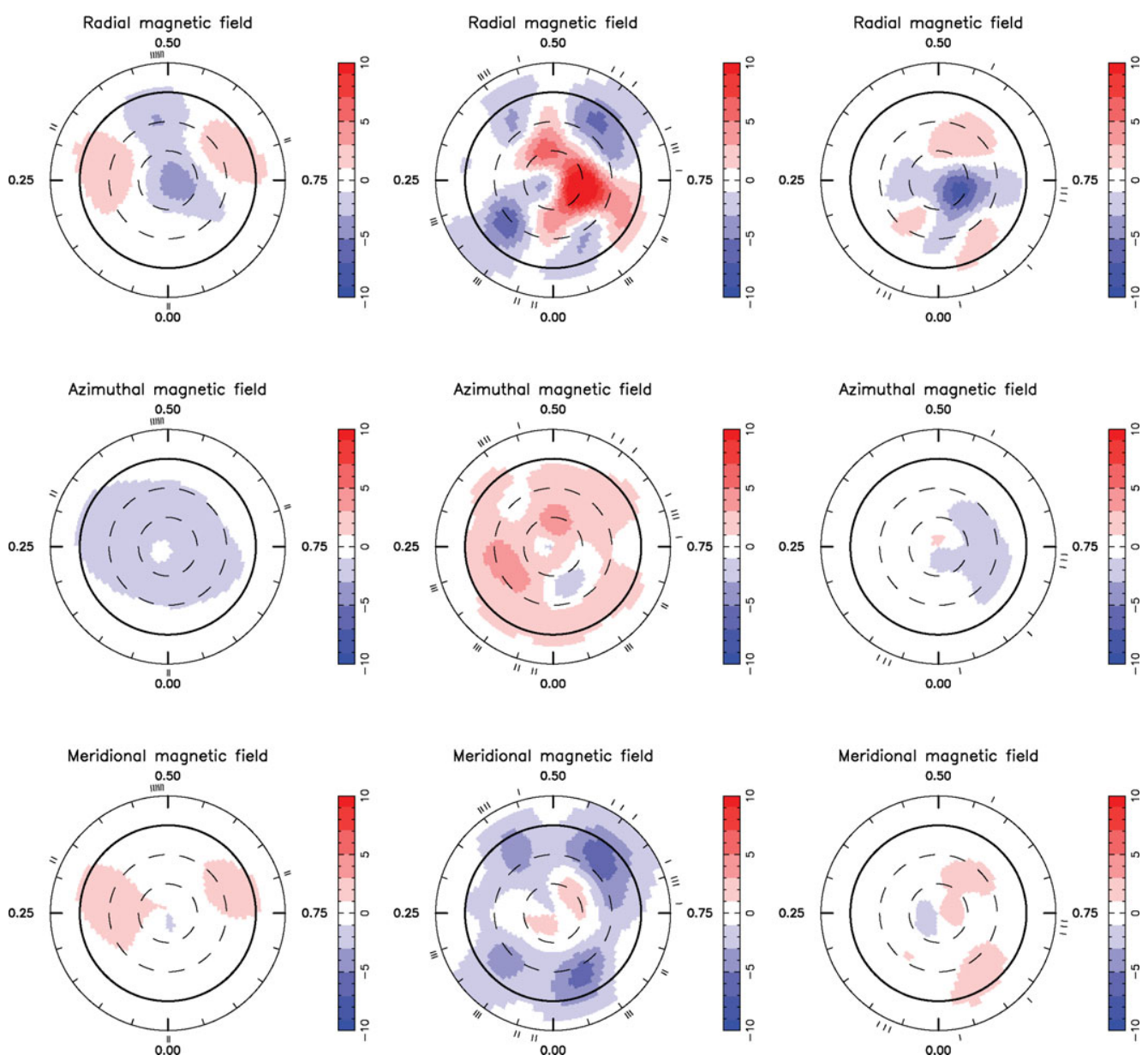

Figure 2. Large-scale magnetic topology of the F7 planet-hosting star $\tau$ Boo derived with Zeeman-Doppler imaging in 2006 June (left panel), 2007 June (middle panel) and 2008 June (right panel). In each panel, the 3 components of the field in spherical coordinates are displayed (from top to bottom) with magnetic fluxes labelled in G, the star being shown in flattened polar projection down to a latitude of $-30^{\circ}$. Radial ticks around each plot indicate phases of observations. Both poloidal and toroidal fields globally switched polarities between successive epochs (from Fares et al. 2009).

predict. The other option, i.e., that these fields would be fossil remnants from a prior evolutionary stage, finds little support either from observations or theory; while models predict fossil fields to be dissipated by convection (as a result of the very high turbulent magnetic diffusivity) in as little as $1000 \mathrm{yr}$, observations indicate that fields are very often highly variable (both locally and globally) on timescales of months to decades and thus cannot reasonably result from an evolutionary process that ended at least tens of Myr before. Magnetic field measurements on cool stars thus bring, at first order, strong and independent support to generic dynamo models.

Dynamo models have undergone considerable progress in recent years; mean-field models are now implementing more physics (e.g., the presence of an interface layer or the effect of meridional circulation) while direct numerical simulations are now able to reach strongly turbulent regimes capable of producing intense mean magnetic fields (Brun et al. 2004, Browning 2008). However, despite such progress, there is still a large number of 
open questions, some of them concerning the very basic physics of dynamo processes, e.g., the identification of the primary mechanism through which the large-scale poloidal component is regenerated (Charbonneau et al. 2005). Above all, dynamo models are almost completely tailored for the Sun, with all model parameters finely tuned to reproduce solar observations as well as possible; checking them against observations of other stars with different masses and rotation rates in particular is a mandatory validation test that they yet have to undergo. The growing body of published results on large-scale magnetic topologies of cool stars should provide the opportunity for doing this in the near future. Meanwhile, we will summarize here the main topics on which the recent results provide new insight into dynamo processes.

The presence of toroidal fields at the surface of partly-convective stars with $R_{o}<1$ is undoubtedly a surprising discovery, leading some to conclude that dynamo processes in very active stars must be operating either throughout the whole CZ or at the very least within a subphotospheric layer (e.g., Donati \& Cameron 2007, Donati 1999, Donati et al. 2003a) rather than just at the base of the CZ (as usually assumed in conventional dynamo theories). Interestingly, a similar idea - a distributed dynamo shaped by nearsurface shear - was recently invoked and investigated theoretically in the particular case of the Sun as an alternative to conventional interface dynamo models (Brandenburg 2005); in particular, this new model can potentially solve a number of long-standing issues (e.g., the large number of toroidal flux belts produced by interface dynamos) if further validated by new simulations. Very recent spectropolarimetric observations of the Sun with the HINODE spacecraft revealed that the quiet inter-network regions (i.e., the inner regions of supergranular cells of the quiet Sun) are pervaded by transient mainly-horizontal magnetic flux (Lites et al. 2008), giving still further support for a nonconventional distributed and/or near-surface dynamo in the Sun. Admittedly, surface toroidal fields detected in cool active stars are more stable than those seen on the Sun and participate to the large-scale field; they could however share a similar origin and scale up in strength and size with $1 / R o$, being only visible at low ZDI-like spatial resolutions for stars with $R o<1$. In turn, this may suggest that the newly-discovered horizontal fields of the Sun also participate to the activity cycle.

Despite their high level of activity, fully-convective stars obviously lack the interface layer where dynamo processes presumably operate; understanding their magnetism thus represents a major challenge for theoreticians. A wide range of predictions has been made about the kind of fields that such dynamos can produce; while early studies speculate that they generate small-scale fields only (e.g., Durney et al. 1993), newer models find that they can potentially trigger purely non-axisymmetric large-scale fields (e.g., Chabrier \& Küker 2006) with CZs rotating as solid bodies (Küker \& Rüdiger 1997). The latest simulations show that axisymmetric poloidal fields can also be produced with significant differential rotation (Dobler et al. 2006), but that toroidal fields are usually dominant and differential rotation rather weak whenever $R o$ is low enough (Browning 2008). In this context, the recent discovery (from both spectropolarimetry and radio observations) that fully-convective stars are able to generate strong and simple large-scale mostlyaxisymmetric poloidal fields while rotating almost as solid bodies (Donati et al. 2006, Morin et al. 2008a, 2008b) is very unexpected and hard to reconcile with any of the existing models.

The first results of the spectropolarimetric survey indicate that the sharp transition in the large-scale magnetic topologies and surface differential rotation of $\mathrm{M}$ dwarfs more or less occurs where the internal structure of the star drastically changes with mass (the inner radiative zone shrinking in radius from $0.5 R_{\star}$ to virtually nothing when $M_{\star}$ decreases from $0.5 \mathrm{M}_{\odot}$ to $0.4 \mathrm{M}_{\odot}$, e.g., Baraffe et al. 1998). It is also worthwhile noting 
that X-ray luminosities of $\mathrm{M}$ dwarfs (relative to their bolometric luminosities) are roughly equal (at similar $R o$ ) on both sides of the full-convection threshold, while the strengths of their large-scale fields feature a clear discontinuity (at a mass of about $0.4 \mathrm{M}_{\odot}$, Donati et al. 2008b, Morin et al. 2008b). All this suggests that dynamo processes become much more efficient at producing large-scale mainly-axisymmetric poloidal fields essentially as a response to the rapid growth in convective depths with decreasing stellar masses; this is qualitatively compatible with the idea that the geometry (and in particular the aspect ratio) of the CZ may control the kind of dynamo wave that a cosmic body can excite (Goudard \& Dormy 2008). The latest finding that dwarfs stars seem capable, at very low-masses and high rotation rates, of generating either strong aligned dipoles or very weak non-axisymmetric large-scale fields (Morin et al. 2010) is equally intriguing, and may suggest a bistable behaviour of dynamo processes in a restricted range of stellar parameters.

The first detection of global magnetic polarity switches in a star other than the Sun is a major first step towards a better understanding of activity cycles of low-mass stars. Looking at, e.g., how cycle periods vary with stellar mass and rotation rate, or how poloidal and toroidal fields fluctuate with time across the cycle period, should ultimately reveal what physical processes mostly control the cycle. Results using a Babcock-Leighton flux transport dynamo model on the Sun suggest that meridional circulation is a crucial parameter (e.g., Dikpati \& Charbonneau 1999); while meridional circulation is difficult to estimate directly in stars other than the Sun, its relation to rotation and differential rotation can potentially be tracked back from how cycle periods vary with stellar parameters. The geometry of the CZ is potentially also important (Goudard \& Dormy 2008).

\section{Conclusion}

Spectropolarimetric observations have the potential to reveal the drastically different magnetic topologies that dynamos of low-mass stars can generate for various stellar masses and rotation rates. They demonstrated in particular that large-scale fields of cool stars change abruptly close to the full-convection threshold, with dwarfs on the low-mass side being much more successful at producing strong, mostly axisymmetric, dipole-like magnetic configurations; partly convective stars also undergo a noticeable change around the $R o=1$ limit. Provided generous allocation of telescope time, spectropolarimetric techniques can even unveil properties of stellar magnetic cycles (and the details of the underlying polarity switches of both poloidal and toroidal field components) as well as how they depend on mass and rotation rates.

Large-scale coordinated observing campaigns organised within the framework of the MagIcS international program (aimed at investigating stellar magnetic fields throughout the HR diagram) should continuously provide new advances along these lines in the future. Extending observations to near-infrared (nIR) wavelengths is of obvious interest, both for reaching very-low-mass stars and brown dwarfs (too faint to be observed in the visible domain) and for improving the overall sensitivity to magnetic fields (thanks to the increased Zeeman splitting); we thus propose to build a new nIR cryogenic spectropolarimeter (called SPIRou) operating from $1-2.5 \mu \mathrm{m}$ as a next-generation instrument for CFHT in 2015.

Time is also ripe for a thorough statistical investigation of theoretical and numerical dynamos; working out in particular whether existing models and codes are able to reproduce convincingly the abrupt changes in the large-scale field properties that observations reveal sounds like an obvious goal for the near future, both potentially fruitful and technically feasible. 


\section{References}

Baraffe I., Chabrier G., Allard F., \& Hauschildt P. H., 1998, A\&A 337, 403

Berdyugina S. V., 2005, Living Reviews in Solar Physics 2, 8

Berger E., Rutledge R. E., Reid I. N., et al., 2005, ApJ 627, 960

Brandenburg A., 2005, ApJ 625, 539

Browning M. K., 2008, ApJ 676, 1262

Brun A. S., Browning M. K., \& Toomre J., 2005, ApJ 629, 461

Cameron A. C. \& Robinson R. D., 1989, MNRAS 236, 57

Chabrier G. \& Küker M., 2006, A\&SA 446, 1027

Charbonneau P., 2005, Living Reviews in Solar Physics 2, 2

Dikpati M. \& Charbonneau P., 1999, ApJ 518, 508

Dobler W., Stix M., \& Brandenburg A., 2006, ApJ 638, 336

Donati J.-F., 1999, MNRAS 302, 457

Donati J.-F., Brown S. F., Semel M., et al., 1992, A\&A 265, 682

Donati J.-F. \& Cameron A. C., 1997, MNRAS 291, 1

Donati J.-F., Cameron A. C., Semel M., et al., 2003a, MNRAS 345, 1145

Donati J.-F., Cameron A. C., \& Petit P., 2003b, MNRAS 345, 1187

Donati J.-F., Forveille T., Cameron A. C., et al., 2006, Science 311, 633

Donati J.-F. \& Landstreet J. D., 2009, ARA $\& A 47,333$

Donati J.-F., Morin J., Petit P., et al., 2008b, MNRAS 390, 545

Donati J.-F., Moutou C., Fares R., et al., 2008a, MNRAS 385, 1179

Donati J.-F., Semel M., Carter B. D., Rees D. E., \& Collier Cameron, A. 1997, MNRAS, 291, 658

Dunstone N. J., Hussain G. A. J., Cameron A. C., et al., 2008, MNRAS 387, 1525

Durney B. R., De Young D. S., \& Roxburgh I. W., 1993, Sol. Phys. 145, 207

Fares R., Donati J.-F., Moutou C., et al., 2009, MNRAS 398, 1383

Goudard L. \& Dormy E., 2008, Europhysics Letters 83, 59001

Hallinan G., Antonova A., Doyle J. G., et al., 2008, ApJ 684, 644

Hall J. C., 2008, Living Reviews in Solar Physics 5, 2

Hall D. S., 1991, in LNP 380, 353 (Berlin Springer Verlag)

Johns-Krull C. M. \& Valenti J. A., 1996, ApJ 459, L95

Küker M. \& Rüdiger G., 1997, A\&\&A 328, 253

Lites B. W., Kubo M., Socas-Navarro H., et al., 2008, ApJ 672, 1237

Mangeney A. \& Praderie F., 1984, A\&SA 130, 143

Marsden S. C., Donati J.-F., Semel M., Petit P. \& Carter, B. D., 2006, MNRAS 370, 468

Mestel L., 1999, Stellar magnetism (Oxford : Clarendon)

Morin J., Donati J.-F., Forveille T., et al., 2008a, MNRAS 384, 77

Morin J., Donati J.-F., Petit P., et al., 2008b, MNRAS 390, 567

Morin J., Donati J.-F., Petit P., et al., 2010, MNRAS (in press)

Noyes R. W., Hartmann L. W., Baliunas S. L., Duncan D. K., \& Vaughan A. H., 1984, ApJ 279, 763

Petit P., Donati J.-F., Aurier̀e M., et al., 2005, MNRAS 361, 837

Petit P., Dintrans B., Solanki S. K., et al., 2008, MNRAS 388, 80

Reiners A., 2006, A\& 4 446, 267

Reiners A. \& Basri G., 2008, ApJ 684, 1390

Robinson R. D., Worden S. P., \& Harvey J. W., 1980, ApJ 236, L155

Saar S. H., 2001, in: ASP Conf Series 223, 292

Schatzman E., 1962, AnAp 25, 18 